\title{
Optimal Coordination of Variable Renewable Resources and Electric Vehicles as Distributed Storage for Energy Sustainability
}

\author{
G. Haddadian, N. Khalili, M. Khodayar, and M. Shahidehpour \\ (ghaddadi@iit.edu, Khalili@iit.edu, mkhodayar@smu.edu, ms@iit.edu)
}

\begin{abstract}
This paper evaluates the coordination between electric vehicle (EV) fleets, as distributed storage devices, and variable renewable sources for mitigating energy imbalances and offering significant potentials for energy sustainability in an electricity infrastructure. The paper investigates the impact of such integrations for enhancing the environmental sustainability, social sustainability, and economic operation of electric power systems. The goal is to keep the energy sector on track for addressing the 2 degree Celsius (2DC) target per Copenhagen climate agreement ${ }^{1}$. The paper identifies strategies for large-scale integration of variable generation resources without compromising the electricity infrastructure security. The power system uncertainties pertaining to hourly load and wind energy forecast errors, and random outages of generation and transmission components are taken into consideration in Monte Carlo scenarios. The stochastic optimization of dayahead hourly scheduling of electricity is formulated as a mixed integer linear programing problem. The merits of the proposed optimization model are demonstrated by applying four numerical case studies. The conclusion is that the applications of renewable energy resources and the intelligent assimilation of EV fleets (both as a provider and a consumer of energy) offer major potentials for alleviating power system peak demands, minimizing power grid operation costs and hourly wind energy curtailments, and limiting the environmental impacts of fossil fuelbased thermal generating units in the stochastic operation of an electricity infrastructure.
\end{abstract}

Keywords - Renewable energy sources, storage, thermal generation emission, stochastic security-constrained unit commitment, V2G.

\section{NOMENCLATURE}

Indices:

$b, j, o:$ Index of bus

$d$ : Index of demand

$i$ : Index of thermal units

$l:$ Index of transmission line

$s:$ Index of a scenario

$t$ : Hour index $(t=1$ to 24$)$

$v$ : Index of EV fleet

$w$ : Index of wind turbine

Variables:

$C_{v, t}^{s}$ : Operation cost of fleet $v$ at time $t$ in scenario $s$ [\$]

$E_{v, t}^{s}$ : Available energy in batteries of fleet $v$ at time $t$ in scenario $s[\mathrm{MWh}]$

$E_{v, t}^{n e t, s}:$ Net discharged energy of EV fleet $v$ at time $t$ in scenario $s$ [MWh]

$F_{c, i}$ : Production cost function of a thermal unit $i$

$F_{e, i}^{E T}:$ Emission function of unit $i$

$F_{c, i}^{r}$ : Availability cost function of a thermal unit $i[\$ / \mathrm{hr}]$

$I_{i, t}:$ Commitment state of unit $i$ at time $t$

$I_{c, \mathrm{v}, \mathrm{t}}^{s}:$ Commitment state of EV fleet $v$ in charging mode at time $t$ in scenario $s$

$I_{d c, v, t}^{s}:$ Commitment state of EV fleet $v$ in discharging mode at time $t$ in scenario $s$

$I_{i, v, t}^{s}:$ Commitment state of EV fleet $v$ in idle mode at time $t$ in scenario $s$

\footnotetext{
1 In 2010, governments agreed that there is an urgent need to reduce emissions so that global temperature escalations are limited to below 2 degrees Celsius.
} 
$M P_{1, t, b}^{S}, M P_{2, t, b}^{S}$ : Non-negative slack variables for real power mismatch at bus $b$ at hour $t$ in scenario $s$

$P_{i, t}^{s}$ : Generation dispatch of a unit $i$ at time $t$ in scenario $s$ [MW]

$P_{d, w, t}:$ Power generation curtailed of wind turbine $w$ at $t[\mathrm{MW}]$

$P L_{l, t}^{s}:$ Real power flow on line $l$ at hour $t$ in scenario $s$ [MW]

$P_{c, v, t}^{s}, P_{d c, v, t}^{s}$ : Charge/discharge power dispatch of EV fleet $v$ at time $t$ in scenario $s$ [MW]

$P_{v, t}^{\varsigma}:$ Generation dispatch of EV fleet $v$ at time $t$ in scenario $s[\mathrm{MW}]$

$P_{m, v, t}^{s}$ : Charge/discharge power dispatch of EV fleet $v$ at segment $m$ at time $t$ in scenario $s$ [MW]

$S D_{i, t}$ : Shutdown cost of a unit $i$ at time $t$ [\$]

$S U_{i, t}$ : Startup cost of a unit $i$ at time $t[\$]$

$W_{t}^{S}$ : Objective function of subproblem

$X_{i t}^{\text {off }}$ : OFF time of unit $i$ at time $t[\mathrm{hr}]$

$X_{i t}^{o n}:$ ON time of unit $i$ at time $t[\mathrm{hr}]$

$y_{i, t}$ : Startup indicator of unit $i$ at time $t$

$z_{i, t}$ : Shutdown indicator of unit $i$ at time $t$

$\Delta_{i t}^{\mathrm{max}}$ : Maximum acceptable power adjustment of a unit $i$ at time $t$ [MW]

$\theta_{j, t}^{s}:$ Bus voltage angle at time $t$ in scenario $s[\mathrm{rad}]$

\section{Parameters:}

$B_{b, t}^{s}:$ Set of units that are connected to bus $b$ at time $t$ in scenario $s$

$b_{m, v}$ : Slope of segment $m$ in linearized charge/discharge curve of EV fleet $v$

$D_{b}$ : Set of loads that are connected to bus $b$

$E_{v}^{\min }, E_{v}^{\max }:$ Min/Max energy stored in batteries of EV fleet $v[\mathrm{MWh}]$

$E M S_{\max }^{E T}$ : Emission limit [lb]

$L_{f, b}, L_{t, b}$ : Set of lines starting from/ending at bus $b$

$N_{v, t}$ : Status of grid connection of fleet $v$ at time $t$

$N E_{v}^{s}$ : Ratio of the number of EVs in fleet $v$ in scenario $s$ to the number of EVs in base case

$N G$ : Total number of units

$N L$ : Total number of lines

$N B$ : Total number of buses

$N T$ : Total number of periods under study

$N V$ : Total number of EV fleets

$P_{c, v}^{\min }, P_{c, v}^{\max }:$ Min/max charging capacity of EV fleet $v[\mathrm{MW}]$

$P_{D, t}$ : Total system demand at time $t[\mathrm{MW}]$

$P_{D, t}^{d, s}:$ Demand served at time $t$ in scenario $s[\mathrm{MW}]$

$P_{d c, v}^{\min }, P_{d c, v}^{\max }:$ Min/max discharging capacity of EV fleet $v$ [MW]

$P_{i, \text { min }}$ : Lower limit of real power generation of unit $i[\mathrm{MW}]$ 
$P_{i, \max }:$ Upper limit of real power generation of unit $i[\mathrm{MW}]$

$P_{m, v}^{\max }:$ Maximum power output at segment $\mathrm{m}$ in charging/discharging cost curve of EV fleet $v$ [MW]

$p^{b}$ : Probability of base case

$p^{s}$ : Probability of scenario $s$

$P L_{l}^{\max }:$ Maximum capacity of line $l[\mathrm{MW}]$

$S D_{e, i t}^{E T}$ : Shutdown emission of unit $i$ at time $t[\mathrm{lb}]$

$S U_{e, i t}^{E T}:$ Startup emission of unit $i$ at time $t[\mathrm{lb}]$

$T_{i}^{o f f}$ : Minimum down time of unit $i[\mathrm{hr}]$

$T_{i}^{o n}$ : Minimum up time of unit $i[\mathrm{hr}]$

$U R_{i}$ : Ramp-up rate of unit $i[\mathrm{MW} / \mathrm{hr}]$

$D R_{i}$ : Ramp-down rate of unit $i[\mathrm{MW} / \mathrm{hr}]$

$U X_{i, t}^{s}$ : Outage status of generation unit $i$ at time $t$ in scenario $s$ (if available 1 , and 0 otherwise)

$U Y_{l, t}^{s}$ : Outage status of the line $l$ at time $t$, in scenario $s$ (if available 1 , and 0 otherwise)

$X_{j \mathrm{o}}$ : Inductance of a line between buses $j$ and $o$ [p.u.]

$\eta_{h}$ : Cycle charging efficiency of EV fleet

$\lambda, \pi, \rho, \mu$ : Lagrangian multipliers

\section{INTRODUCTION}

Economic, social, and environmental challenges will have to be balanced as the electric utility industry progresses toward a more sustainable and modern grid, while meeting its principal obligations of delivering affordable, reliable, and safe electricity to its consumers. According to the International Energy Agency (IEA) [1], the electricity industry is not on track to limit the long-term escalation in the average global temperature to 2 degrees Celsius (2DC) per the Copenhagen Accord. As such electric utilities face formidable challenges for satisfying energy triangle obligations pertaining to environmental sustainability, energy security, and economic competitiveness. As the world looks to combat climate changes, a shift from carbon-based fuels to noncarbon based fuels is inevitable. At the same time, distributed generating units are employed progressively at load centers for promoting energy efficiency, alleviating the dependence on foreign fossil fuel, and boosting the security of transmissionconstrained power systems. In this regard, sustainability is shifted from a nice-to-do to a must-to-do paradigm in the electric utility industry which requires pioneering technologies for addressing energy challenges [2].

It is possible to considerably reduce carbon footprints in electric power systems through large-scale integrations of renewable resources. Given proper scales, clean and renewable energy has the potential to support the balance of the energy triangle. According to IEA, the increased share of power generation from renewables, as well as natural gas in tandem with limited use of least efficient coal-fired plants, would curb emissions by $640 \mathrm{Gt}$ in 2020 and help restrain local air pollutions. According to the World Wind Energy Association (WWEA) [3], the total installed global wind energy capacity increased from $18 \mathrm{GW}$ to $175 \mathrm{GW}$ between 2000 and 2010 . However, while traditionally large thermal power plants can be operated as base power supply, and many can ramp up and down to address electricity demand fluctuations, the variability of renewable sources can disturb the balance of electricity generation and demand.

The large integration of wind energy (greater than $30 \%$ of energy) into the electricity infrastructure necessitates the redesign of conventional power systems and the modification of their operating practices [4]. Although an increase in the number and the geographic distribution of wind turbines can alleviate the temporal variability of wind energy and reduce wind speed forecasting errors, the lack of seasonal correlation of wind energy and electricity demand could result in energy balance disparities [5], [6]. Several methods can be adopted to address this challenge which include the integration of flexible thermal plants (e.g. natural gas turbines), expansion and assimilation of transmission grid, demand response practices, and the utilization of energy storage ranging from batteries, ultra-capacitors, compressed-air storage, flywheels to hydroelectric storage reservoirs [7], [8].

Batteries, with the possibility of fast charging and discharging, can play a significant role among storage alternatives. The surplus wind energy can be stored in batteries and discharged at a later time when needed. Thus, a battery storage system can by capture the wind energy surplus during off peak periods when the demand is low, and improve the reliability of energy supply during peak hours when the marginal generation cost is high [9]-[11]. Additional benefits of battery include load leveling, 
spinning reserve, area regulation, long line stabilization, power factor correction and black start capability [12],[13]. However, an efficient battery control system is required to optimize battery life issues and minimize the associated maintenance costs. So, adding storage for mitigating the wind energy variability will further increase the operating cost of wind energy system [14],[15].

Electric vehicle (EV) batteries, among storage alternatives offer numerous options for the large-scale integration of variable renewable energy. Vehicle-to-Grid (V2G) facilitates a bi-directional energy flow between EVs and the electricity infrastructure, in which the storage can withdraw power from the electricity grid for charging at low price hours and inject power back to the grid during peak hours when electricity prices are high. The coordinated V2G system is viewed as an attractive multi-mode generating unit which may be used for mitigating transmission flow congestions, lowering operating costs by shutting down peaking units, minimizing the variability of renewable energy sources, and reducing system emissions.

The V2G effect on generation costs [16],[17], transmission [18], and distribution transformer loading [19],[20] is investigated by a number of studies. The system-wide generation efficiency is enhanced if an EVs' charge/discharge pattern is properly coordinated with the dispatch of cleaner and more efficient generating units [21]-[24]. V2G can also reduce the operating cost plug-in hybrid electric vehicle (PHEV) as compared with that of conventional vehicles [21]. [22] examines the use of PHEV fleet in both controlled and uncontrolled scenarios which result in lowering the gasoline consumption as compared to internal combustion engine (ICE) vehicles in the state of Ohio. Moreover, the regulatory framework within which PHEVs are deployed plays a major role in determining the net emission yield from PHEVs charged in power systems. A Texas study demonstrated that imposing power system emission constraints would shift most of the PHEV charging from coal to cleaner natural gas-fired units at no additional generation costs because of the synergy between unit commitment (UC) and PHEV charging controls [23].[24] investigates charging decisions under a variety of electricity tariffs with a non-convex UC optimization algorithm. The results indicate that constraining mid-day EV charging is less important than charging PHEVs at late evening hours.

Although at present, the EV market is limited, it is projected that the market will flourish with advances in battery technologies. For the purpose of this study, EVs are considered stationary as various studies indicate that most vehicles are parked for an average of $90 \%$ of the time [25], [26]. Furthermore, the concept of EV mobility could be minor with respect to the power system size. In this context, EV fleets are not traversing between system buses as distances between buses are assumed relatively large in this paper. In essence, EVs remain within the domain of a given bus and connected to the power network during the EV operation period. In addition, EV fleets are aggregated both as supply and demand at each bus.

This paper proposes a new and practical scheduling methodology for EVs that has the potential to improve sustainability strategies in the energy sector and keep the sector on track to address the 2DC climate goals by 2050 while addressing energy security issues. The paper discusses the modeling of aggregated EV fleets as stationary distributed load and energy storage facilities in the power system infrastructure, while considering the coordinated and large-scale integration of wind energy for limiting fossil fuel emissions. The paper examines the synergy between the storage and renewable energy sources for limiting the carbon footprint and minimizing the operation cost of security-constrained power systems. Hourly load and wind energy forecast errors, as well as random outages of generation and transmission components are also taken into consideration in this study.

The contributions of this paper include, 1) modeling EV fleets as distributed energy storage facilities in an electricity infrastructure; 2) coordinated scheduling of EV fleet charging/discharging by considering the economic and the environmental impacts of such schedules on power system operations; 3) presenting uncertainties in the power system operation scheduling and addressing economic and environmental challenges imposed by such uncertainties; and 4) developing a decomposed framework based on the Benders decomposition (BD) technique to address the security of power systems in the base case and prevailing Monte Carlo scenarios. The optimization of the stochastic day-ahead hourly security-constrained unit commitment (SCUC) is formulated as a mixed integer linear programing (MILP) problem. Furthermore, considering the large-scale nature of the coordination problem, BD is applied to tackle this problem in real-time. The deployment of BD reduces the complexity of the optimization problem by decomposing the original large-scale MILP problem into one mixed integer program (MIP) master problem and several linear programing (LP) sub-problems. An iterative process between the master problem and sub-problems delivers a minimized cost of generation scheduling while taking into account network and emission constraints.

The remaining of this paper is structured as follows. The proposed stochastic SCUC optimization model is described in Section III. The simulation outcomes emphasizing the effectiveness of the proposed model are presented and discussed in Section IV. Finally, Section V sums up the core results and conclusions of the study and offers some insights on potential future developments of the proposed method.

\section{Proposed Day-Ahead Scheduling}

The proposed day-ahead scheduling problem coordinates variable energy sources, mainly wind energy, with stationary EV fleets, as distributed storage facilities. The proposed approach demonstrates how this coordination can effectively satisfy electricity infrastructure requirements while achieving economic goals with substantial cutback on carbon-footprints. Furthermore, the proposed model is applied to optimize the hourly coordination of wind energy-EV fleet generation with the thermal unit dispatch. The proposed solution applies BD and utilizes a de power flow model to address network constraints. 


\section{A. Stochastic Security-Constrained Unit Commitment}

Electric utilities benefit in terms of their operation costs from the optimal UC and economic dispatch (ED) of thermal generation units. Short-term UC will outline the hourly on/off status of thermal units over a day while considering the cost and addressing physical constraints of thermal units. ED will determine the least cost operation of a power system by dispatching available generation resources to supply the system hourly load, while satisfying operation constraints. Optimization techniques such as Lagrangian relaxation (LR), and MILP are most widely applied to UC and ED for solving day-ahead and real-time generation scheduling problems [27]-[31]. SCUC is a non-deterministic polynomial-time hard (NP-hard), non-convex, nonlinear, mixed-integer optimization problem with a large number of binary scheduling variables, continuous and discrete control variables, and a series of prevailing equality and inequality constraints [32]. The MILP method obtains a better solution than that of LR in a finite number of steps. This feature facilitates broader applications of the MILP method in electricity markets. Moreover, it is easier to add constraints to the MILP model and achieve an optimal solution, without involving heuristics, which can dramatically accelerate the development of UC and facilitate its applications to large-scale electricity infrastructure systems [32]. The main drawback of MILP is its computational complexity when applied to large-scale SCUC problems. However, powerful algorithms such as branch-and-cut and commercial solvers with potentials for large-scale applications can soften the computation burden of MILP.

\section{B. Stochastic SCUC formulation}

In this study, the coordination of wind energy, stationary EV fleets, and conventional thermal units is formulated as a MILP problem in stochastic SCUC. The objective (1) is to minimize the power system operation cost, in which load and wind energy forecasts are scheduled subject to the system and generating unit constraints.

$$
\left\{\min \left[\sum_{t} \sum_{i}\left(p^{b} F_{c, i}\left(P_{i, t}\right)+S U_{i, t}+S D_{i, t}\right)+\sum_{t} \sum_{v} p^{b} \cdot C_{v, t}\right]+\left[\sum_{t} \sum_{i}\left(F_{c, i}^{r}\left(\Delta_{i, t}^{\max }\right)\right)\right]+\sum_{s} p^{s} \cdot\left[\sum_{t} \sum_{i} F_{c, i}\left(P_{i, t}^{s}\right)+\sum_{t} \sum_{v} C_{v, t}^{s}\right]\right\}
$$

where the production cost function expressed as a quadratic function of the power dispatch,

$F_{c, i}\left(P_{i, t}\right)=a_{c, i}+b_{c, i} \cdot P_{i, t}+c_{c, i} \cdot P_{i, t}^{2}$ in which $a, b$, and $c$ are the cost coefficients.

The objective function (1) consists of the base case operation cost, including the generation and startup/shutdown costs of thermal units, and the operation cost of EV fleets, in which case the outages of generators and transmission lines are not included. Further, availability costs to generators for facilitating spinning reserves in multiple scenarios are taken into consideration as the second term in the objective function. One third of the marginal cost of a generating unit is considered as the availability cost [33]. In response to uncertainties, the provision of reserve is exercised as a remedial action by generators. The generators' remedial actions are bound by ramp up/down limitations. The objective function also considers the expected cost of remedial actions in scenarios for accommodating uncertainties. Several models are available for considering the startup cost $(S U)$ and the shutdown cost $(S D)$ which reflect the number of hours a unit has been off and on, respectively. For the sake of simplicity, startup and shutdown costs are considered fixed which are imposed once the unit is turned on or off. Thermal units are assumed to be non-quick start units and, as such, their commitment status in scenarios is the same as that in the base case. So no startup/shutdown costs are introduced in scenarios. The expected operation cost in scenarios is shown as the third term in (1). The nonlinear production cost function is approximated by a set of piecewise linear blocks as is shown in Fig. 1. The operation cost of EVs, $C_{v, t}$, depends on the number of vehicles and the charging/discharging depth and frequency [34]. Further, $F_{c, i}^{r}\left(\Delta_{i, t}^{\max }\right)$ represents the hourly cost of corrective action.

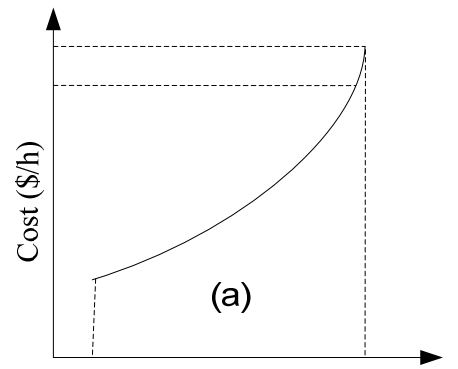

Power(MW)

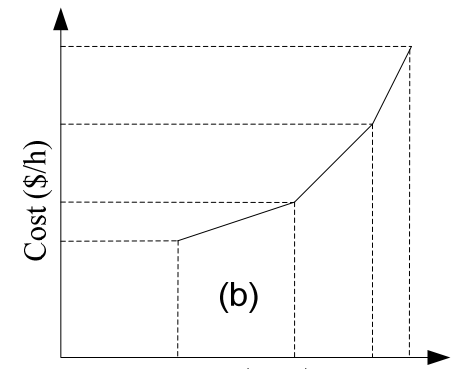

Power(MW)

Fig.1. Linearization (a) Nonlinear Curve, (b) Piecewise Linear Curve

The proposed formulation for thermal and wind generation units (2)-(11) are developed in the base case by ignoring the scenario indices to provide a clear description of constraints. Similar formulations apply to scenarios. It should be noted that the commitment states of thermal units are not dependent on scenarios. The wind energy curtailment happens when there is either an inadequate ramping down capability of thermal units or a major transmission congestion for utilizing the available wind energy in electricity infrastructures. The wind energy curtailment constraint is given in (2) in which the sum of dispatched and curtailed wind energy is the same as the wind energy forecast. The thermal unit generation range (3) implies that once the unit is 
committed $(I=1)$, the generation unit must operate between its minimum and maximum generating capacity. If $I=0$, the unit is de-committed and its generation dispatch is zero. The system load and demand balance is shown in (4). The minimum off/on time constraints for each unit are shown in (5) and (6) respectively. The minimum off time indicates the number of hours that a unit must be off before it can be turned on again. Similarly, the minimum on time is the number of hours that a unit must be on before it can be turned off. The unit ramp up/down limits between adjacent hours are demonstrated by (7)-(8). Here, (7) conveys that when unit $i$ starts up at time $t$, its generation dispatch $\left(P_{i, t}\right)$ is equal to the minimum generating capacity of unit $\left(P_{m i n}\right)$, while

(8) shows when unit $i$ is shuts down at time $t$, its generation dispatch $\left(P_{i(t-1)}\right)$ is equal to the minimum generating capacity of unit $\left(P_{\min }\right)$ [33]. If the unit is on in two consecutive hours, the generation dispatch is limited by the ramping up/down limits shown in (7) and (8). Here if the unit stays on, the binary variables representing startup/shutdown states are set to zero and the dispatch in two consecutive hours is limited by ramping up/down limits. The relationship between startup/shutdown indicators and the commitment status of units is shown in (9). Constraint (10) shows that the startup/shutdown states of a unit are mutually exclusive. Constraint (11) indicates that the daily emission is capped. For the purpose of this study, the primary emission concerns are carbon dioxide $\left(\mathrm{CO}_{2}\right)$ and carbon dioxide equivalent $\left(\mathrm{CO}_{2 \mathrm{e}}\right)$. The emission function, as a convex quadratic function of power generation, is modeled $\mathrm{as}^{2}: F_{e, i}\left(p_{i t}\right)=\alpha_{e i}+\beta_{e i} p_{i t}+\gamma_{e i} p_{i t}^{2}$ where $\alpha_{e i}, \beta_{e i}, \gamma_{e i}$ represent emission coefficients ${ }^{3}$ of $^{2}$ unit $i$.

$$
\begin{aligned}
& P_{w, t}+P_{d, w, t}=P_{f, w, t} \\
& P_{i, \min } I_{i, t} \leq P_{i, t} \leq P_{i, \max } I_{i, t} \quad \forall i, \forall t \\
& \sum_{i=1}^{N G} P_{i, t}+\sum_{w=1}^{N W} P_{w, t}+\sum_{v=1}^{N V} P_{v, t}=P_{D, t} \quad[t=1, \ldots, N T] \\
& {\left[X_{i(t-1)}^{o f f}-T_{i}^{o f f}\right] *\left[I_{i t}-I_{i(t-1)}\right] \geq 0 \quad \forall i, \forall \mathrm{t}} \\
& {\left[X_{i(t-1)}^{o n}-T_{i}^{o n}\right] *\left[I_{i(t-1)}-I_{i t}\right] \geq 0 \quad \forall i, \forall \mathrm{t}} \\
& P_{i t}-P_{i(t-1)} \leq P_{i, \min } \cdot y_{i t}+\left(1-y_{i t}\right) \cdot R U_{i} \\
& P_{i(t-1)}-P_{i t} \leq P_{i, \min } \cdot z_{i t}+\left(1-z_{i t}\right) \cdot R D_{i} \\
& I_{i t}-I_{i(t-1)}=y_{i t}-z_{i t} \\
& y_{i t}+z_{i t} \leq 1 \\
& \sum_{i}^{2} \sum_{t}^{24}\left[F_{e, i}^{E T}\left(P_{i t}\right)+S U_{e, i t}^{E T}+S D_{e, i t}^{E T}\right] \leq E M S_{\max }^{E T} \quad E T=\left\{C O_{2} \& C O_{2 e}\right\}
\end{aligned}
$$

Emission constraints are coupling constraints over a group of generating units and periods of study. The startup/shutdown emissions denoted by $S U_{e, i t}^{E T}$ and $S D_{e, i t}^{E T}$. The nonlinear emission function is piecewise linearized and incorporated into the proposed MILP formulation. The system and EV fleet constraints in (12)-(25) are shown in scenarios. The system and generating unit constraints in Monte Carlo scenarios are similar to those in the base case constraints. The system power balance and dc power flow constraints are expressed by (12)-(13), respectively. The grid connection of EV fleet at time $t$ is illustrated by $B_{b, t}^{v}$ in (12). The power flow constraint (13) indicates that transmission flows are dependent on corresponding incremental bus voltage angles and line impendences. Here, a transmission line flow is limited by (14) and set to zero as the line is on outage. Constraint (15) addresses the scenario's corrective action which indicates any dispatch deviation from the base case requires corrective actions performed by available generation units. The constraint corresponding to an unavailable unit in a scenario would be relaxed so that the corrective action would not be considered by the generation unit. The scenario constraints for EV fleets are demonstrated in (16)-(24) where (16) conveys the net hourly absorbed/injected energy and the dispatched power of EV fleet. Here, the difference between the energy stored in the aggregated EV battery and the EV energy injected back to the grid is measured by the charging cycle efficiency of the aggregated EV. An EV fleet connected to the power grid $\left(N_{v, t}=1\right)$, will be in charged, discharged, or the idle mode (17). The limitations on EV charging/discharging imposed by power electronic interfaces and charging stations are shown in (18) and (19). Here, EV charging/discharging in each scenario is dependent on connectivity $\left(N_{v, t}\right)$ and the number of EVs $\left(N E_{v}^{s}\right)$. The hourly energy balance is ensured by (20). The fleet energy range is addressed in (21) representing the EV fleet capacity limit. It is assumed in (22) that the state of charge of EV fleets at the end of the operation

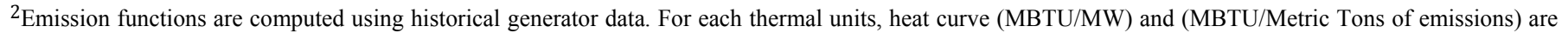

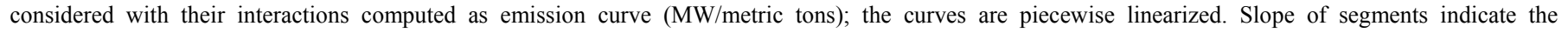
incremental emission for each unit.
} 
period is the same that of the initial period. The nonlinear battery charging/discharging cost curves which are convex quadratic functions are piecewise linearized in the MILP formulation. A tighter piecewise linear estimation is presented in [34]. The piecewise linear convex charge/discharge cost curve of EV batteries is expressed by (23) which shows a direct correlation with the number of EVs and the depth of charging/discharging cycles. A higher in-depth battery charging/discharging causes the number of cycles to failure decrease dramatically, which corresponds to a higher cost of EV charging/discharging [35]. The aggregated state of charge (SOC) of batteries is fixed at specific operation periods in a consumer-controlled scenario as stated by (24). The base case constraints are similar to (16)-(24) considering $N E_{v}^{S}=1$. The expected emission limit for emission constraints in a scenario is shown in (25).

$$
\begin{aligned}
& \sum_{i \in B_{b}^{i}} P_{i, t}^{S}+\sum_{v \in B_{b, t}^{v}} P_{v, t}^{S}+\sum_{w \in B_{b}^{w}} P_{w, t}^{S}=\sum_{d \in D_{b}} P_{D, t}^{d, s}+\sum_{l \in L_{f, b}} P L_{l, t}^{S}-\sum_{l \in L_{t, b}} P L_{l, t}^{S} \\
& -M .\left(1-U Y_{l, t}^{s}\right) \leq P L_{l, t}^{s}-\left(\frac{\theta_{j, t}^{s}-\theta_{o, t}^{s}}{X_{j o}}\right) \leq M .\left(1-U Y_{l, t}^{s}\right) \\
& -P L_{l}^{\max } U Y_{l, t}^{s} \leq P L_{l, t}^{s} \leq P L_{l}^{\max } U Y_{l, t}^{s} \\
& \left\{\begin{array}{l}
-\Delta_{i t}^{\max } \leq P_{i t}^{S}-U X_{i t}^{S} \cdot P_{i t} \leq \Delta_{i t}^{\max } \\
P_{i}^{\min } \cdot U X_{i t}^{S} \cdot I_{i t} \leq P_{i t}^{S} \leq P_{i}^{\max } \cdot U X_{i t}^{S} \cdot I_{i t}
\end{array}\right. \\
& \left\{\begin{array}{l}
E_{v, t}^{n e t, s}=\eta_{v} \cdot P_{c, v, t}^{s}-P_{d c, v, t}^{s} \\
P_{v, t}^{s}=P_{c, v, t}^{s}-P_{d c, v, t}^{s}
\end{array}\right. \\
& I_{d c, v, t}^{s}+I_{c, v, t}^{s}+I_{i, v, t}^{s}=N_{v, t} \\
& N_{v, t} \cdot I_{c, v, t}^{s} \cdot P_{c, v}^{\min } \cdot N E_{v}^{s} \leq P_{c, v, t}^{s} \leq N_{v, t} \cdot I_{c, v, t}^{s} \cdot P_{c, v}^{\max } \cdot N E_{v}^{s} \\
& N_{v, t} \cdot I_{d c, v, t}^{s} \cdot P_{d c, v}^{\min } \cdot N E_{v}^{s} \leq P_{d c, v, t}^{s} \leq N_{v, t} \cdot I_{d c, v, t}^{s} \cdot P_{d c, v}^{\max } \cdot N E_{v}^{s} \\
& E_{v, t}^{S}=E_{v, t-1}^{S}-E_{v, t}^{n e t, s} \\
& E_{v}^{\min } . N E_{v}^{S} \leq E_{v, t}^{s} \leq E_{v}^{\max } . N E_{v}^{S} \\
& E_{v, 0}^{S}=E_{v, N T}^{S}=E_{v 0} \cdot N E_{v}^{S} \\
& \left\{\begin{array}{l}
C_{v, t}^{s}=N_{v, t} \cdot\left[\sum_{m} b_{m, v} \cdot P_{m, v, t}^{S}\right] \quad 0 \leq P_{m, v, t}^{s} \leq P_{m, v}^{\max } \cdot N E_{v}^{s} \\
N_{v, t} \cdot\left|E_{v, t}^{S}-E_{v, t-1}^{S}\right|=\sum_{m} P_{m, v, t}^{S}
\end{array}\right. \\
& E_{v, \widehat{T}}^{s}=E_{v}^{\max } \cdot N E_{v}^{s} \\
& \sum_{i} \sum_{t}^{24}\left\{p^{b} \cdot\left[F_{e, i}^{E T}\left(P_{i t}\right)+S U_{e, i t}^{E T}+S D_{e, i t}^{E T}\right]+\sum_{s} p^{s} \cdot\left[F_{e, i}^{E T}\left(P_{i t}^{s}\right)+S U_{e, i t}^{E T}+S D_{e, i t}^{E T}\right]\right\} \leq \operatorname{expected}\left(E M S_{\max }^{E T}\right)
\end{aligned}
$$

The flowchart of the proposed stochastic SCUC formulation is depicted in Fig. 2. The two-level hierarchical structure of the proposed MILP problem makes it a suitable candidate for BD. Indeed, decomposition is the only feasible option for the solution of a large-scale stochastic SCUC problem in real time [36]. Considering the stochastic nature and the practical size of the SCUC problem, which involves hundreds/thousands of generating units, transmission lines, and study hours, BD offers a proper tool for decomposing the original large-scale MILP problem into one integer program master (MIP) problem and several linear programing (LP) sub-problems.

The master problem of BD solves the hourly UC with dominant constraints. The lower bound solution of the master problem involves fewer constraints. The sub-problems of BD will examine the dc power flow according to the master problem's UC solution in the base case and scenarios for minimizing transmission flow violations. Transmission networks, which are assessed independently for the base case and scenarios, are optimized in parallel. If any violation arises, the corresponding feasibility cuts will be generated and added to the master problem for the solution of the next iteration. Accordingly, a new lower bound solution of the original problem will be attained by re-calculating the master problem with the additional constraints. The process continues until all violations are mitigated. The optimal solution of the original problem will occur when upper and lower bounds are adequately close, which will confine the final solution to be close to the global optimal solution of the original problem [36]. The iterative process between the master problem and sub-problems delivers a minimized cost solution for 
generation scheduling while addressing transmission, voltage, and emission constraints. Here the master problem is formed in (1)-(11) with similar constraints for scenarios in (15)-(25), while the sub-problem is shown in (26)-(31) and (13),(14) for each scenario. Note that a similar formulation can be written for base case variables ignoring the scenario index and setting the availability of generation units and transmission lines to one. Once the solution of master problem is passed on to the subproblem (for either a base case or a scenario), the nodal power balance is checked for any violations considering the line flow limitations. If there is any violation in the solution of master MILP problem at any iteration, Benders cut (32) for each scenario and time step is formed and sent back to the master problem. The master problem is solved again with the new constraint and this iterative process continues until no violation exists (i.e. the objective function in (26) becomes zero).

The proposed stochastic UC is a multi-stage stochastic programming problem. The first set of decision variables are determined before uncertainties are realized (here and now decisions) while the second set of variables are determined after the uncertainties are realized (wait and see decisions). The here and now decisions represent the generation unit commitment while the wait and see decisions are generation dispatch quantities.

\begin{tabular}{|c|c|}
\hline $\operatorname{Min} W_{t}^{s}\left(\hat{I}_{t}, \hat{P}_{t}^{s}\right)=\sum_{b}\left(M P_{1, t, b}^{s}+M P_{2, t, b}^{S}\right)$ & (26) \\
\hline \multicolumn{2}{|l|}{ s.t. } \\
\hline$\sum_{i \in B_{b}^{i}} P_{i, t}^{S}+\sum_{w \in B_{b}^{w}} P_{w, t}^{S}+\sum_{v \in B_{b, t}^{v}} P_{v, t}^{S}+M P_{1, t, b}^{S}-M P_{2, t, b}^{S}=\sum_{d \in D_{b}} P_{D, t}^{d, s}+\sum_{l \in L_{f, b}} P L_{l, t}^{S}-\sum_{l \in L_{t, b}} P L_{l, t}^{S}$ & $(27)$ \\
\hline$M P_{1, t, b}^{S}, M P_{2, t, b}^{S} \geq 0$ & $(28)$ \\
\hline$P_{i, t}^{S}=\hat{P}_{i, t}^{s}$ & $(29)$ \\
\hline$P_{v, t}^{S}=\hat{P}_{v, t}^{s}$ & $(30)$ \\
\hline$P_{w, t}^{s}=\hat{P}_{w, t}^{s} \quad \mu_{w, t}^{s}$ & (31) \\
\hline$W_{t}^{s}\left(I_{t}, P_{t}^{s}\right)=W_{t}^{s}\left(\hat{I}_{t}, \hat{P}_{t}^{s}\right)+\sum_{i} \pi_{i, t}^{s}\left(P_{i, t}^{s}-\hat{P}_{i, t}^{s}\right)+\sum_{v} \lambda_{v, t}^{s}\left(P_{v, t}^{s}-\hat{P}_{v, t}^{s}\right)+\sum_{w} \mu_{w, t}^{s}\left(P_{w, t}^{s}-\hat{P}_{w, t}^{s}\right) \leq 0$ & $(32)$ \\
\hline
\end{tabular}

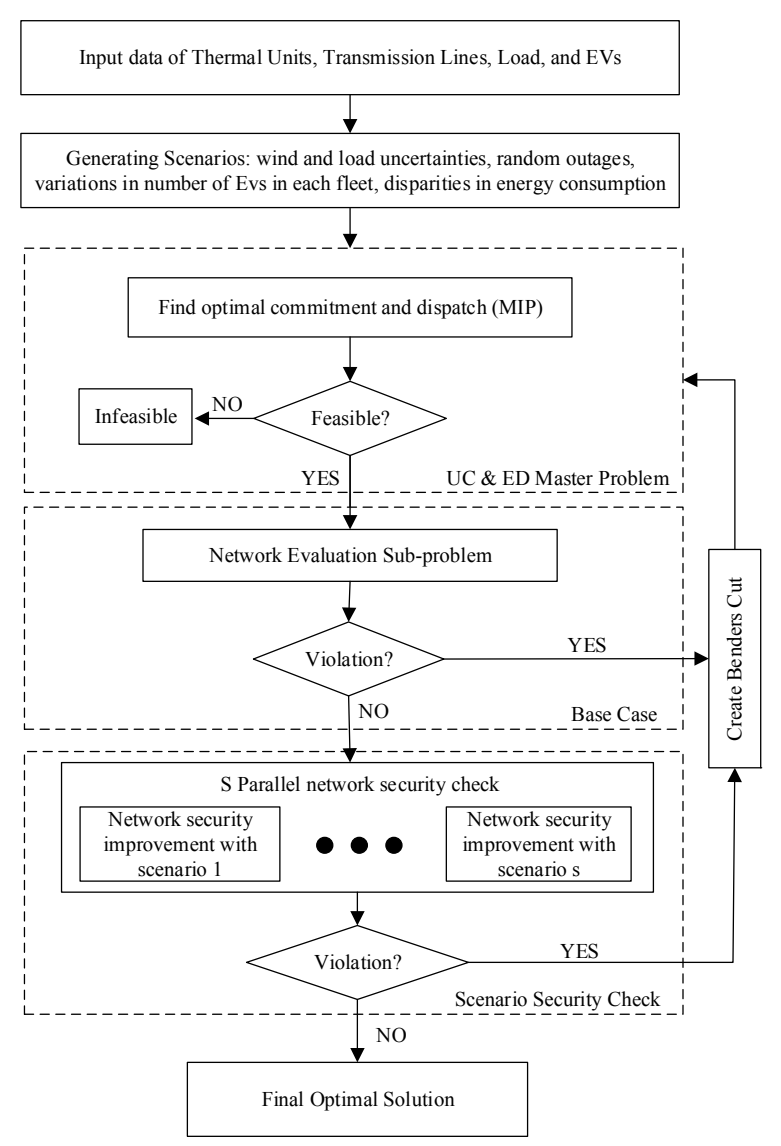

Fig. 2. The Proposed Stochastic SCUC for the Coordinated Scheduling of Constrained Thermal, EV, and Renewable Energy Units 


\section{CASE STUdies}

In this section, a 6-bus power system shown in Fig. 3 is utilized to demonstrate the effectiveness of the proposed day-ahead scheduling solution for EV fleets. The examples investigate the coordination of wind energy-EV fleets at a bus-level and the system-level on the hourly commitment and dispatch of coal and natural gas units. Furthermore, the total expected operation cost, base cost, capacity cost, total diurnal expected emission, base and expected wind energy curtailment are evaluated in the following cases.

\section{A. System Topology}

The 6-bus system incorporates two coal units (G1,G2), one natural gas unit (G3), and a wind turbine. G1-G3 units are the least to the most expensive units, which are also the most to the least pollutant, respectively. Furthermore, the system includes 7 transmission lines. The parameters of generating units, transmission lines, and hourly load forecasts for 24-h are depicted in Tables I and II and Fig. 4, respectively. Table III shows the emission function coefficients.

The installed wind unit capacity is $75 \mathrm{MW}$, which is about $30 \%$ of the power system peak load. In addition, the Monte Carlo simulation is deployed for incorporating power system uncertainties such as random outages of generators and transmission lines, and forecast errors of hourly loads and wind speeds. Forced outage rates (FOR) of power system components and distribution functions for load and wind speed forecast errors comprise parameters in Monte Carlo simulations. Here, transmission line and generation unit FOR are listed as $1 \%$ and $4 \%$, respectively. Equation (33) denotes the load forecast error by a truncated normal distribution function in which the mean value is the hourly power forecast and the standard deviation is $5 \%$ of the mean [37].

$$
\begin{aligned}
& f(x)=\left\{\begin{array}{l}
0 \\
\frac{1}{\alpha \sqrt{2 \pi} \sigma} \cdot e^{-\frac{(x-\mu)^{2}}{2 \sigma^{2}}} \quad \mu-3.5 \sigma \leq x \leq \mu+3.5 \sigma
\end{array}\right. \\
& \text { where } \alpha=\int_{\mu-3.5 \alpha}^{\mu+3.5 \alpha} \frac{1}{\sqrt{2 \pi} \sigma} \cdot e^{-\frac{(x-\mu)^{2}}{2 \sigma^{2}}} d x, \mu \text { and } \sigma \text { are the mean and the standard deviation of the distribution function, }
\end{aligned}
$$

respectively. The wind speed forecast error is demonstrated by the auto-regressive moving average (ARMA) model [38],[39]. As the time lag increases, the autocorrelation factor (ACF) and the partial autocorrelation factor (PACF) will be lower, and a lower order ARMA $(1,1)$ represented in (34) will be deployed. The ARMA constants are obtained by minimizing the difference in the root mean square error (RMSE) between the simulated ARMA model and the measured wind speed [39]. In this paper, it is assumed that the ARMA constants are $\alpha=0.98$ and $\beta=-0.7$. The standard deviation of the Gaussian distribution function is $10 \%$ of the wind speed forecast for a 1-hour lag time. The forecasted wind speed time series is obtained by implementing the probability transition matrix, which is procured either by historical data or by probability distribution parameters of wind speed [40]. Wind speed is categorized into several groups represented by the mean value at each group. The probability transition matrix outlines probabilities for transiting from one wind speed category to others. The probability transition matrix is secured by composing an initial probability vector, a weighting matrix, and a normalizing vector, through the deployment of the Weibull distribution function and the autocorrelation factor [40]. Once the probability transition matrix is constructed, the wind speed time series will be obtained by the Markov chain method [41]. The Markov chain method is deployed to synthesize the wind speed time series consistent with the procured probability transition matrix [40]-[41].

$$
X(t)=\alpha \cdot X(t-1)+\beta \cdot Z(t-1)+Z(t)
$$

The diurnal pattern strength which denotes the daily wind speed pattern with sinusoidal form is applied to time series. The hourly wind energy is calculated consistent with the power curve of wind turbines and the hourly wind speed. A low discrepancy method, Latin Hypercube Sampling (LHS), is developed to obtain evenly distributed random samples with a smaller variance for improving the efficiency of scenario-based stochastic simulation [42]-[44]. Additionally, scenario reduction techniques attain a goodness-of-fit tradeoff between computation speed and results accuracy by excluding scenarios with very low probabilities and bundling scenarios that are very close in terms of statistical metrics [42]. Potential scenario reduction algorithms consist of fast backward, fast forward, and fast backward/backward methods [45]-[46]. The algorithms have various computation performances subject to the problem size and the required solution precision. For large scenario trees, the fast backward method delivers the best computation performance with the worst precision. The results of the fast forward method are more precise, but the computational time is longer. In this paper, the fast backward/forward method is adopted to reduce the number of scenarios. 


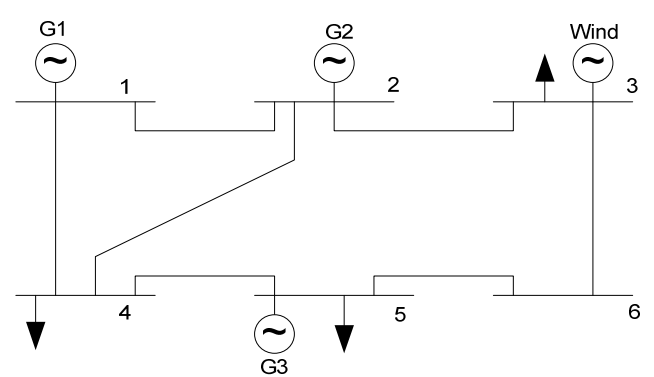

Fig. 3. Six Bus power system

TABLE I

THERMAL UNIT CHARACTERISTICS

\begin{tabular}{c|c|c|c|c|c|c|c|c|c}
\hline Unit & $\begin{array}{c}\mathrm{a} \\
\left(\$ / \mathrm{MW}^{2}\right)\end{array}$ & $\begin{array}{c}\mathrm{b} \\
(\$ / \mathrm{MW})\end{array}$ & $\begin{array}{c}\mathrm{c} \\
(\$ / \mathrm{h})\end{array}$ & $\begin{array}{l}\mathrm{P}_{\min } \\
(\mathrm{MW})\end{array}$ & $\begin{array}{l}\mathrm{P}_{\max } \\
\mathrm{MW})\end{array}$ & $\begin{array}{c}\text { SU } \\
(\$)\end{array}$ & $\begin{array}{c}\text { SD } \\
(\$)\end{array}$ & $\begin{array}{c}\text { Min. } \\
\text { Up }(\mathrm{h})\end{array}$ & $\begin{array}{c}\text { Min } \\
\text { Dn. (h) }\end{array}$ \\
\hline $\mathrm{G} 1$ & 0.099 & 6.589 & 211.4 & 100 & 320 & 100 & 50 & 4 & 3 \\
\hline $\mathrm{G} 2$ & 0.203 & 7.629 & 217.4 & 10 & 160 & 200 & 40 & 3 & 2 \\
\hline $\mathrm{G}^{\mathrm{C}}$ & 0.089 & 6.58 & 210.4 & 10 & 220 & 10 & 80 & 1 & 1 \\
\hline $\mathrm{G}^{\mathrm{G}}$ & 0.494 & 10.07 & 102.8 & 10 & 100 & 80 & 10 & 1 & 1 \\
\hline
\end{tabular}

TABLE II

TRANSMISSION LINE CHARACTERISTICS

\begin{tabular}{c|c|c|c|c}
\hline Line ID & $\begin{array}{c}\text { From } \\
\text { Bus }\end{array}$ & $\begin{array}{c}\text { To } \\
\text { Bus }\end{array}$ & $\begin{array}{c}\text { Impedance } \\
\text { (p.u) }\end{array}$ & $\begin{array}{c}\text { Capacity } \\
(\mathrm{MW})\end{array}$ \\
\hline 1 & 1 & 2 & 0.17 & 122 \\
\hline 2 & 1 & 4 & 0.258 & 160 \\
\hline 3 & 2 & 4 & 0.197 & 80 \\
\hline 4 & 5 & 6 & 0.14 & 96 \\
\hline 5 & 3 & 6 & 0.018 & 160 \\
\hline 6 & 2 & 3 & 0.037 & 160 \\
\hline 7 & 4 & 5 & 0.037 & 96 \\
\hline
\end{tabular}

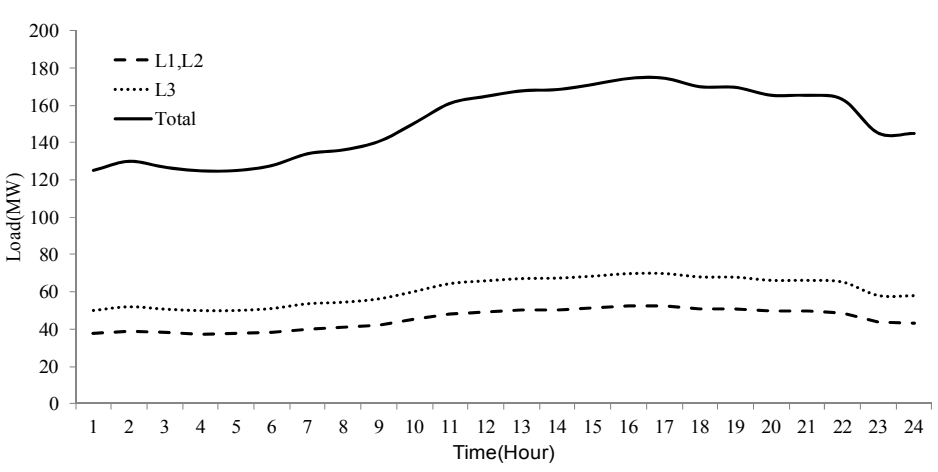

Fig. 4. Hourly Load

TABLE III

EMISSION FUNCTION COEFFICIENTS

\begin{tabular}{cccc}
\hline Unit & $\begin{array}{c}\alpha \\
\left(\mathrm{LbCO}_{2} / \mathrm{MWh}^{2}\right)\end{array}$ & $\begin{array}{c}\beta \\
\left(\mathrm{LbCO}_{2} / \mathrm{MWh}\right)\end{array}$ & $\begin{array}{c}\gamma \\
\left(\mathrm{LbCO}_{2} / \mathrm{h}\right)\end{array}$ \\
\hline $\mathrm{G} 1$ & 0.000304 & 19.943 & 0.0 \\
\hline $\mathrm{G} 2$ & 0.000312 & 18.933 & 0.0 \\
\hline $\mathrm{G}^{\mathrm{C}}$ & 0.000300 & 17.934 & 0.0 \\
\hline $\mathrm{G}^{\mathrm{G}}$ & 0.000351 & 10.032 & 0.0 \\
\hline
\end{tabular}

\section{B. Case Studies}

The following 4 cases are examined in which the diurnal emission cap of 86,300 pounds and diurnal expected emission cap of 175,000 pounds is imposed in all cases. Daily emissions are computed using historical generator data. Similarly, daily emission cap is imposed based on the historical data. The optimization problem is solved using CPLEX 12.5.0 on a 2.3 GHz intel Core i7 with an $8 \mathrm{~GB}$ of memory.

Case 1: Stochastic SCUC with two coal units and one natural gas unit considering environmental externalities

Case 2: Stochastic SCUC with two coal units, one natural gas unit, and one wind turbine considering environmental externalities Case 3: Intelligent integration of stationary EV fleets and wind energy, and their coordination in the hourly stochastic SCUC solution considering environmental externalities.

Case 4: Rule-based integration of stationary EV fleets and wind energy and their coordination in the hourly stochastic SCUC 
solution considering environmental externalities.

The proposed cases are discussed as follows:

Case 1: In this case, the coordination between 2 coal units (G1, G2) and one gas unit (G3) is evaluated without considering any variable renewable sources. In Case 1, load forecast errors and generation, and transmission outages are considered. The load forecast error follows a normal distribution with the predicted load as the mean value and a standard deviation that is $5 \%$ of the mean value. Table IV illustrates the hourly UC in which all units are committed for 24 hours. The daily base cost, availability cost, and expected cost are \$121,445, \$10,762, \$121,991.695 respectively. Fig. 5 depicts the diurnal generation dispatch of thermal units, in which G1 dispatches more power to minimize the cost, while the other coal unit (G2) dispatches less as compared to the natural gas unit (G3) to address the emission constraints. In Fig. 6, G1 emits the most followed by G2, and G3, respectively. The cumulative expected daily emission in scenarios is 73,789.270 lb. This case has 13,081 variables including 12,862 continuous variables, and 219 integer variables; and the solution time is 10.64 seconds.

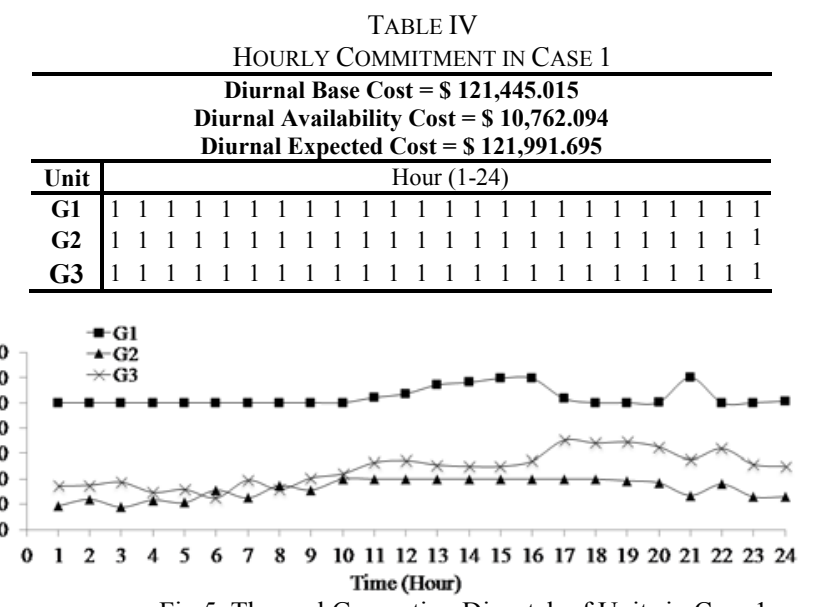

Fig.5. Thermal Generation Dispatch of Units in Case 1

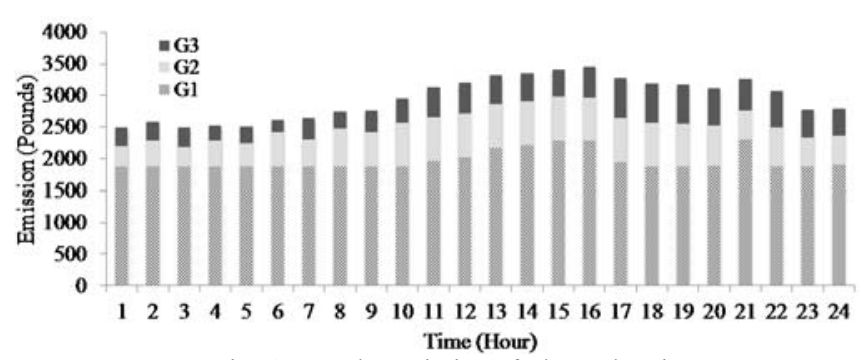

Fig. 6. Hourly Emission of Thermal Units - Case 1

Case 2: In this case, the coordination between 2 coal units (G1, G2), one gas unit (G3), and one renewable energy source (wind turbine) is examined. Wind energy forecast errors are considered and the wind unit generation is based on the wind speed data and typical wind power curve. The mean daily wind speed is $10 \mathrm{~m} / \mathrm{s}$, which follows a Weibull distribution function with a coefficient that is equal to 2.1. In Table V, G1 and G2 are committed for 24 hours while G3, which is the most expensive gas unit, is committed during peak hours for minimizing the cost. The sufficient ramping down capability of the natural gas unit supports the variable wind energy source. The daily base cost, availability cost, and expected cost drop by $16.91 \%, 31.92 \%$, and $20.07 \%$ to $\$ 100,913.29, \$ 7,326.04$, and $\$ 97,501.83$, respectively. The natural gas unit which serves as a peak load supplier will adjust its dispatch within minutes, which will offer a potential for the integration of variable energy sources. The wind energy curtailment in the base case and the expected wind energy curtailment in scenarios are $377.93 \mathrm{MWh}$ and 107.30 MWh. Fig. 7 portrays the diurnal generation dispatch of thermal units in which the standard deviations of generation dispatch for G1, G2, and G3 are $13.15,7.15$, and $10.15 \mathrm{MW}$, respectively. Fig. 8 shows that G2 and G3 discharge less emission as compared to the previous case. The wind turbine is followed closely by natural gas, which reduces the cumulative expected daily emission in scenarios by $8.91 \%$ to $67,775.51 \mathrm{lb}$. This case has 13,405 variables including 13,186 continuous variables, and 219 integer variables; with a solution time of 5.91 seconds.

TABLE V

UNIT COMMITMENT IN CASE 2

\begin{tabular}{|c|c|c|c|c|c|c|c|c|c|c|c|c|c|c|c|c|c|c|c|c|}
\hline \multicolumn{21}{|c|}{$\begin{array}{c}\text { Diurnal Base Cost }=\$ 100,913.293 \\
\text { Diurnal Availability Cost }=\$ 7,326.035 \\
\text { Diurnal Expected Cost }=\$ 97,501.829\end{array}$} \\
\hline Unit & & & & & & & & & & & $(1-2)$ & & & & & & & & & \\
\hline G1 & 1 & 1 & & & 1 & 1 & 1 & 1 & 1 & 1 & 11 & 1 & 1 & 1 & & 1 & & & 1 & 11 \\
\hline G2 & 1 & 1 & & & 1 & 1 & 1 & 1 & 1 & 1 & 11 & 1 & 1 & 1 & 1 & 1 & 1 & 1 & 1 & 1 \\
\hline G3 & 0 & & & 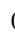 & 1 & 1 & 1 & 1 & 1 & 1 & 1 & 11 & 1 & 1 & 1 & 0 & 0 & & & 0 \\
\hline
\end{tabular}




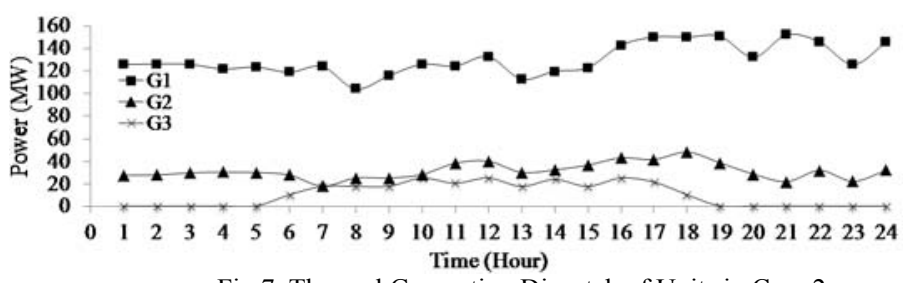

Fig.7. Thermal Generation Dispatch of Units in Case 2

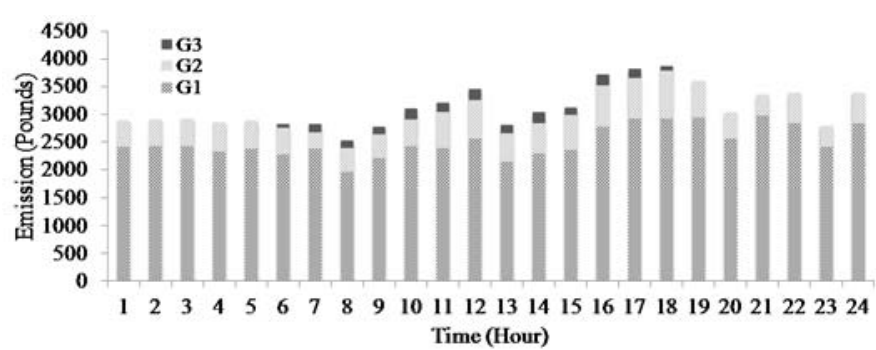

Fig. 8. Hourly Emission of Thermal Units - Case 2

In Cases 3 and 4, two modes of operation are considered for the V2G deployment. In the intelligent-controlled mode, power system operators control the EV fleets charge/discharge decisions based on the system operation requirements. While in the rule-based mode, SOCs are tuned at certain hours to showcase consumer charging/discharging adjustments.

Case 3 - Intelligent-controlled V2G: In order to further optimize the operation cost, abate the emission level, and facilitate higher integrations of variable renewable energy resources, we introduce 5 stationary EV fleets, as distributed storage, that are always connected to a specific bus. It is assumed that EVs are connected to the grid through level II charging stations. The power capacity of residential and commercial level II charging stations are 3.8-7.2 kW and 7.2-16.8 $\mathrm{kW}$ correspondingly [47]. Here the charging and discharging power capacity for each EV is assumed as 7.3 and $6.2 \mathrm{~kW}$, respectively. The battery capacity is $19 \mathrm{kWh}$ for each EV in the first fleet and $27.4 \mathrm{kWh}$ in the second through the third fleet. The EV fleet characteristics include $\mathrm{max} / \mathrm{min}$ capacities, SOC, and charge/discharge capacities of aggregated vehicles. Table VI denotes the characteristics of the three EV fleets consisting of 3,400, 4000, and 2,600 vehicles, respectively.

Table VII illustrates the hourly UC schedule of generators in which the G3 commitment is reduced by four hours at peak. The V2G introduction in power systems could reduce the diurnal base cost, availability cost and expected cost by $5.41 \%$, $63.48 \%$, and $6.29 \%$ to $\$ 95,457.61, \$ 2,675.73$, and $\$ 91,372.30$. Further, the base case and the expected wind energy curtailment in scenarios are ameliorated to $210.77 \mathrm{MWh}$ and $90.51 \mathrm{MWh}$ correspondingly.

Fig. 9 shows the generation dispatch profile which indicates that the standard deviations for the dispatch of G1, G2, and G3 are reduced to $7.88,2.79$, and $10.83 \mathrm{MW}$, respectively; implying a smoother generation profile as compared to that in Case 2 . The generation dispatch volatility could increase the grid operation cost caused by turbine wear and tear. As such, a flexible EV control can moderate the volatility when integrating renewable resources into the grid. Fig. 10 demonstrates the stacked daily diurnal emission dispatch of generators is declined to $67,322.37 \mathrm{lb}$ as compared to the previous cases due to a wider usage of wind energy and the EV storage at peak hours. This case has 36,870 variables including 31,971 continuous variables, and 4,899 integer variables; with a solution time of 191.5 seconds.

TABLE VI

\begin{tabular}{c|c|c|c|c|c|c|c|c}
\multicolumn{10}{c}{ EV FLEET FEATURES } \\
$\begin{array}{c}\text { EV } \\
\text { fleet } \\
\text { No. }\end{array}$ & $\begin{array}{c}\text { Bus } \\
\text { No. }\end{array}$ & $\begin{array}{c}\text { Min } \\
\text { Cap. } \\
(\mathrm{MWh})\end{array}$ & $\begin{array}{c}\text { Max } \\
\text { Cap. } \\
(\mathrm{MWh})\end{array}$ & $\begin{array}{c}\text { Min } \\
\text { Charge/ } \\
\text { Discharge } \\
(\mathrm{kW})\end{array}$ & $\begin{array}{c}\text { Max } \\
\text { Charge/ } \\
\text { Discharge } \\
(\mathrm{MW})\end{array}$ & $\begin{array}{c}\mathrm{a} \\
\left(\$ / \mathrm{MWh}^{2}\right)\end{array}$ & $\begin{array}{c}\mathrm{b} \\
(\$ / \mathrm{MWh})\end{array}$ & $\begin{array}{c}\mathrm{c} \\
(\$ / \mathrm{h})\end{array}$ \\
\hline 1 & 3 & 13.152 & 65.76 & $7.3 / 6.2$ & $24.8 / 21.08$ & 0.17 & 8.21 & 0 \\
\hline 2 & 4 & 21.92 & 109.6 & $7.3 / 6.2$ & $29.16 / 24.8$ & 0.20 & 8.21 & 0 \\
\hline 3 & 5 & 14.248 & 71.24 & $7.3 / 6.2$ & $18.96 / 16.12$ & 0.31 & 8.21 & 0 \\
\hline
\end{tabular}

TABLE VII

UNIT COMMITMENT IN CASE 3

\begin{tabular}{|c|c|c|c|c|c|c|c|c|c|c|c|c|c|c|c|c|c|c|}
\hline \multicolumn{19}{|c|}{$\begin{array}{c}\text { Diurnal Base Cost }=\$ 95,457.611 \\
\text { Diurnal Availability Cost }=\$ 2,675.725 \\
\text { Diurnal Expected Cost }=\$ 91,372.304\end{array}$} \\
\hline Unit & & & & & & & & & & our ( & $(1-24$ & & & & & & & \\
\hline G1 & 1 & & 1 & 1 & 1 & 1 & 1 & 1 & 1 & 11 & 11 & 1 & 1 & 11 & & & 11 & $\begin{array}{ll}1 & 1\end{array}$ \\
\hline G2 & 1 & & 1 & 1 & 1 & & 11 & 1 & 1 & $\begin{array}{ll}11 & 1\end{array}$ & 11 & 1 & 11 & 11 & 1 & 1 & & 11 \\
\hline G3 & 0 & & & & & & & & & 11 & & & & & & & & \\
\hline
\end{tabular}




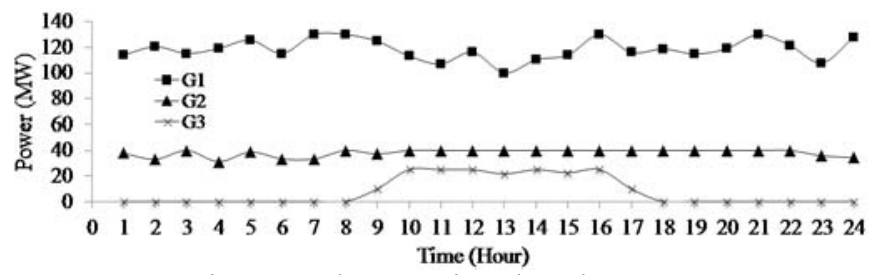

Fig. 9. Hourly Generation Dispatch - case 3

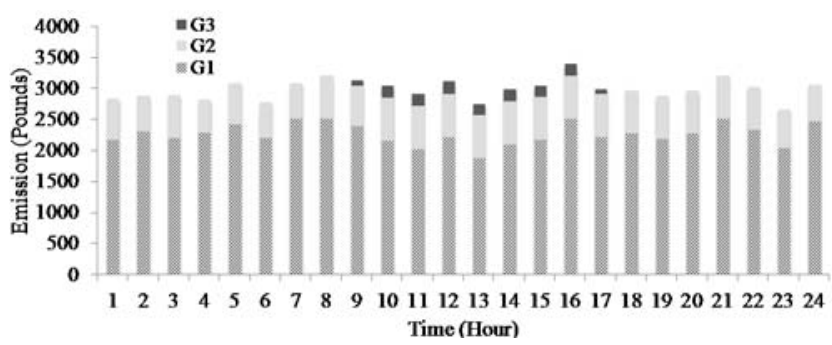

Fig. 10. Hourly Emission of Thermal Units - case 3

Case 4 - Rule-based V2G: In this case, consumers enforce further constraints on EV charging/discharging. As such, the base case operation, availability, and expected costs are increased, in comparison with those in Case 3, to $\$ 96,097.86, \$ 4,273.43$, and $\$ 93,084.26$, respectively. In addition, the base case wind energy curtailment and its expected values in scenarios are 222.91 $\mathrm{MWh}$ and $90.18 \mathrm{MWh}$, respectively; here, the base case wind energy curtailment is increased as compared with that in the previous case. Table VIII displays the hourly UC in Case 4 in which the G3 commitment is decreased by one hour. In Fig. 11, the standard deviations of generation dispatch for G1-G3 are increased to $11.11,5.71$, and $10.10 \mathrm{MW}$, indicating sharp disparities in the generation profile of G1 and G2 in comparison with those in the previous case. Fig. 12 shows the aggregated daily emission dispatch of generators in which the diurnal aggregated expected emission is slightly higher than that in the previous case, which is due to less efficient use of EV fleets.

Table IX summarizes the optimal diurnal base case cost, wind energy curtailment, and aggregated emission in each case. Fig. 13 shows the aggregated hourly load dispatch in the base case, with and without the optimal V2G deployment. At off-peak hours when LMPs ${ }^{4}$ are lower, the EV fleets are either charged or in the idle mode, and as such the demand is higher. At hour 10 when the LMP increases at peak hours, EV fleets would supply power back to the power system which would lower the aggregated demand. So the distributed storage through $\mathrm{V} 2 \mathrm{G}$ could accommodate the variability of renewable energy resources for supplying the hourly demand. Fig.14 illustrates that the wind generation/utilization in Cases 3 and 4 is higher than that in Case 2. Once again the example confirms the positive impact of distributed storage on the integration of renewable sources. In Cases 3 and 4, the use of stationary EV fleets as distributed storage faciliates a smoother integration of wind energy in which a smaller amount of wind energy is curtailed as compared to that in Case 2. Also as Fig.14 indicates, the wind energy curtailment is reduced in Case 3 as compared to that in Case 4 which is due to a more efficient charging/discharging decision for EV fleets. This case has 36,870 variables including 31,971 continuous variables, and 4,899 integer variables; with a solution time of 172.6 seconds.

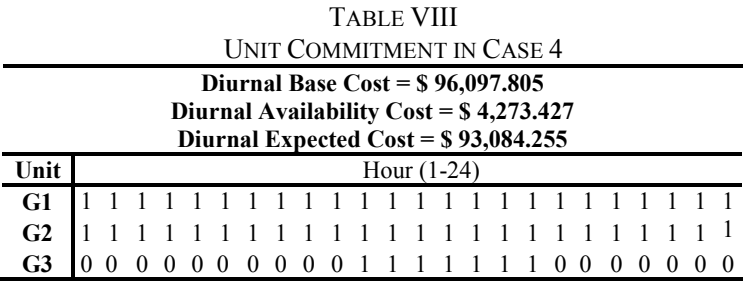

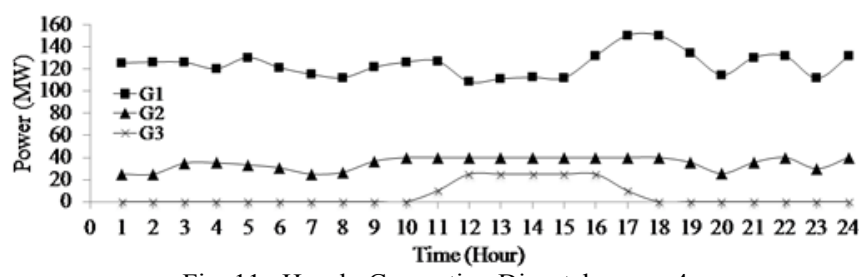

Fig. 11. Hourly Generation Dispatch - case 4

${ }^{4}$ LMP is the Locational Marginal Price which expresses the hourly price at each bus. 


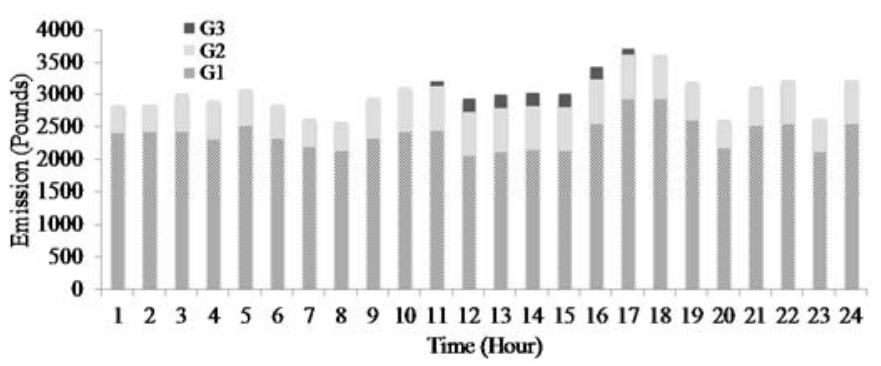

Fig. 12. Hourly Emission of Thermal Units - case 4

TABLE IX

SUMMARY OF RESULTS FOR FOUR CASES (BASE CASE)

\begin{tabular}{c|c|c|c}
\hline Case & Cost $(\$)$ & Wind Curtailment $(\mathrm{MWh})$ & Emission $\left(\mathrm{LbCO}_{2}\right)$ \\
\hline 1 & $121,445.06$ & NA & $73,789.270$ \\
2 & $100,913.29$ & 377.93 & $67,775.510$ \\
3 & $95,457.61$ & 210.765 & $67,322.365$ \\
4 & $96,097.86$ & 222.914 & $67,767.739$ \\
\hline
\end{tabular}

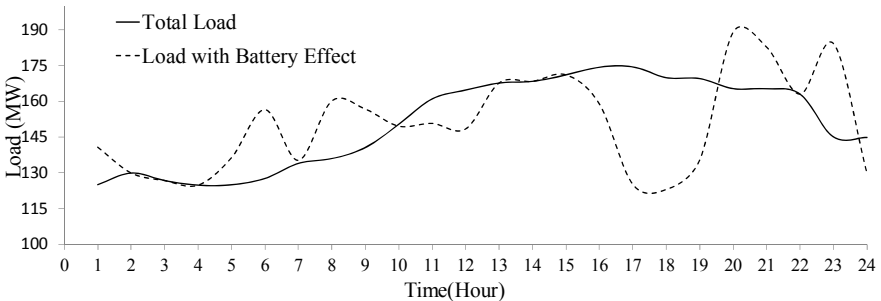

Fig. 13. Hourly Aggregated Load with and without storage (Base Case)

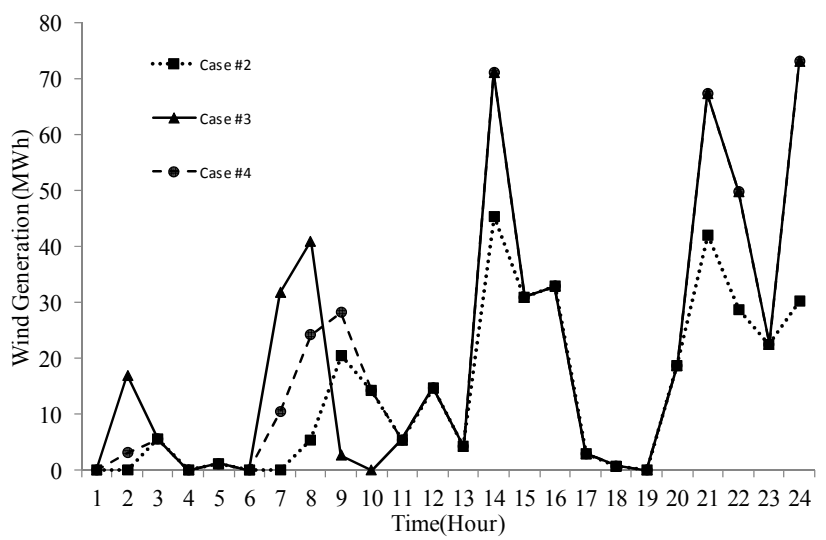

Fig. 14. Hourly Wind Generation/Utilization with and without Storage (Base Case)

\section{CONCLUSIONS}

This paper offers a stochastic programming framework for the day-ahead scheduling of EVs in the electricity infrastructure. The coordinated infrastructure considers uncertainties in the system operation pertaining to wind energy, power grid, EV fleets as distributed storage, and thermal units. The proposed optimization algorithm considers economic, environmental, operation security merits of the day-ahead scheduling. Furthermore, the flexibility embedded in the proposed model makes it suitable for the V2G implementation in practical power systems. Accordingly, the coordination between EV fleets and variable renewable sources provides the electricity sector with a practical tool to spearhead environmental, social, and economic pillars of sustainability. The proposed algorithm also provides potentials for lowering the gap between the world's trend for energy supply and a pathway that is harmonious with the 2DC climate goal. Hence, this paper offers a decision-making process that would facilitate a more effective transition to new energy supplies and deliveries and gauge the impact of introducing EV fleets as virtual power plants and an essential element of a comprehensive energy policy. The simulation results in this paper indicate that the optimal scheduling of EV fleets as stationary distributed storage facilities can cut diurnal operation costs, abate fossil fuel emissions, and enable superior daily wind energy consumption profiles with minimal hourly wind energy curtailments. The optimal V2G implementation is an especially promising method for ensuring that the renewable energy supply would match the hourly demand, smooth out the variability of resources, and provide a long-term, decentralized form of electricity storage in electricity infrastructures. Although distributed storage systems are much smaller than conventional energy sources for 
providing a firm capacity, they demonstrate advantageous technical and economical features when providing short-term power supplies. Further, our analyses point out that the V2G technology has the potential of enforcing a paradigm shift in a number of ways we fundamentally operate electric power systems, which include delaying the installation of conventional peak generation capacity, encouraging the installation of renewable electricity sources, and accelerating the adaptation of electric transportation technologies. An expansion of the proposed model for considering the EV mobility would demonstrate a progressive reduction in regional fossil fuel emissions when conventional vehicles with combustion engines are replaced gradually with EV fleets.

\section{REFERENCES}

1. International Energy Agency (IEA)

Redrawing the Energy-Climate Map World Energy Outlook 2013 special report

http://www.iea.org/publications/freepublications/publication/WEO_RedrawingEnergyClimateMap.pdf, (2003)

2. World Economic Forum (WEF) in partnership with Accenture

New Energy Architecture; Enabling an effective transition

http://www3.weforum.org/docs/WEF_NewEnergyArchitecture IndustryAgenda.pdf, (2012)

3. World Wind Energy Association (WWEA) Information/ Statistics

http://www.wwindea.org/

4. T. Ackermann, L. Söder

Wind power in power systems: an introduction

John Wiley \& Sons Ltd, (2005), pp. 25-52.

5. D. Harvey

Energy and the new reality 2-carbon-free energy supply

1st ed. London: Earthscan, (2010)

6. H. Lund, W. Kempton

Integration of renewable energy into the transport and electricity sectors through V2G

Energy Policy, Vol. 36, No. 9, (2008), pp. 3578-3587.

7. P. Denholm, M. Hand

Grid flexibility and storage required to achieve very high penetration of variable renewable electricity

Energy Policy, Vol. 39, Issue 3, (2011), pp. 1817-1830

8. W. Tian, M. Shahidehpour, Z. Li

Analysis of 2030 Large-Scale Wind Energy Integration in the Eastern Interconnection Using WINS

The electricity Journal, Vol. 24, (2011), pp.71-87

9. G.N. Bathurst, G. Strbac

Value of combining energy storage and wind in short-term energy and balancing markets

Electric Power Systems Research 67, (2003), pp.1-8

10. M. Black, G. Strbac

Value of storage in providing balancing services for electricity generation systems with high wind penetration Journal of Power Sources 162, (2006), pp. 949-953

11. Korpaas, M., Holen, A. T., Hildrum, R.

Operation and sizing of energy storage for wind power plants in a market system

International Journal of Electrical Power \& Energy Systems 25, (2003), pp. 599-606.

12. SK. Aditya, D. Das

Battery energy storage for load frequency control of an interconnected power system

Electric Power Systems Research, Vol. 58, (2001), pp.179-185.

13. I. Momber, A. Siddiqui, T. G. San Román, and L. Söder,

Risk averse scheduling by a PEV aggregator under uncertainty

IEEE Trans. Power Syst. vol. 30, no. 2, pp. 882-892, March 2015.

14. M. Khalid, AV. Savkin

A model predictive control approach to the problem of wind power smoothing with controlled battery storage Renewable Energy, Vol. 35, (2010), pp. 1520-1526

15. R. Sioshansi

Increasing the Value of Wind with Energy Storage

The Energy Journal 32, (2011), pp.1-30

16. P. Denholm and W. Short

An Evaluation of Utility System Impacts and Benefits of Optimally Dispatched Plug-In Hybrid Electric Vehicles

National Renewable Energy Laboratory, Tech. Rep. NREL/TP-620- 40293, (2006)

17. M. Kintner-Meyer, K. Schneider, and R. Pratt

Impacts Assessment of Plug-in Hybrid Vehicles on Electric Utilities and Regional US Power Grids: Part 1: Technical Analysis

Journal of EUEC, vol. 1, (2007) 
18. L. Wang, A. Lin, and Y. Chen

Potential impacts of recharging plug-in hybrid electric vehicles on locational marginal prices

Naval Research Logistics, vol. 57, (2010)

19. K. Clement-Nyns, E. Haesen, and J. Driesen

The Impact of Charging Plug-In Hybrid Electric Vehicles on a Residential Distribution Grid

IEEE Transactions on Power Systems, vol. 25, pp. 371-380, (2010)Q.

20. Gong, S. Midlam-Mohler, V. Marano, and G. Rizzoni

PEV Charging Impact on Residential Distribution Transformer Life

in 2011 IEEE Energytech. Cleveland, OH, United States: Institute of Electrical and Electronics Engineers, (2011)

21. R. Sioshansi and P. Denholm

The value of plug-in hybrid electric vehicles as grid resources

The Energy Journal, vol. 31, pp. 1-23, (2010)

22. R. Sioshansi, R. Fagiani, and V. Marano

Cost and emissions impacts of plug-in hybrid vehicles on the Ohio power system

Energy Policy, vol. 38, (2010)

23. R. Sioshansi and J. Miller

Plug-in hybrid electric vehicles can be clean and economical in dirty power systems

Energy Policy, vol. 39, pp. 6151-6161, (2011)

24. R. Sioshansi

Modeling the Impacts of Electricity Tariffs on Plug-in Hybrid Electric Vehicle Charging, Costs, and Emissions, Operations Research, vol. 60, pp. 506-516, (2012)

25. J. Wang, C. Liu, et al (2011)

Impact of plug-in hybrid electric vehicles on power systems with demand response and wind power

Energy Policy, Vol. 39, (2011), pp. 4016-4021

26. S. Wirasingha, N. Schofield, A. Emadi

Plug-in hybrid electric vehicle developments in the US: trends, barriers, and economic feasibility

IEEE Vehicle Power and Propulsion Conference (VPPC), (2008)

27. M. Carrion, M. José, Arroyo

A computationally efficient mixed-integer linear formulation for the thermal unit commitment problem

IEEE Trans. Power Syst. 21 (2006), pp.1371-1378

28. D. Streiffert, R. Philbrick, A. Ott

A mixed integer programming solution for market clearing and reliability analysis

IEEE PES General Meeting, June (2005)

29. X. Li, T. Li, J. Wei, G. Wang, W.W.G. Yeh

Hydro unit commitment via mixed integer linear programming: a case study of the three gorges project, China IEEE Trans. Power Syst. 29, (2014)

30. G.W. Chang, Y.D. Tsai, C.Y. Lai, J.S. Chung

A practical mixed integer linear programming based approach for unit commitment

IEEE PES General Meeting, June (2004)

31. S. Virmani, E.C. Adrian, K. Imhof, S. Mukherjee

Implementation of a Lagrangian relaxation based unit commitment problem

IEEE Trans. Power Syst. 4 (1989), pp. 1373-1380

32. M. Shahidehpour, T. Li (2005)

Price-based unit commitment: A case of Lagrangian relaxation versus mixed integer programming

IEEE Trans. Power Syst., Vol. 20, No. 4, (2005), pp. 2015-2025

33. D. Gan, E. Litvinov

Energy and reserve market design with explicit consideration to lost opportunity costs

IEEE Trans. Power Syst., Vol. 18, no. 1, (2003), pp. 53-59

34. M. Khodayar, L. Wu, M. Shahidehpour

Hourly coordination of electric vehicle operation and variable wind power generation in SCUC

IEEE Transactions on smart grid, Vol. 3, No. 3, (2012)

35. J. Tomić, W. Kempton

Using fleets of electric-drive vehicles for grid support

Journal of Power Sources, Vol. 168, No. 2, (2007), pp. 459-468

36. M. Shahidehpour, Y. Fu

Benders decomposition

37. IEEE Power and Energy Magazine, Vol. 3, No. 2, (2005), pp. 20-21B. Hodge, A. Florita, K. Orwig, D. Lew, and M.

Milligan,

A comparison of wind power and load forecasting error distributions

2012 ld Renewable Energy Forum, May 2012. 
38. R. Bo and F. Li, Impact of load forecast uncertainty on LMP

Proc. Power System Conference and Exposition, pp. 1-6, Mar. 2009.

39. M. A. Ortega-Vazquez and D. S. Kirschen,

Economic impact assessment of load forecast errors considering the cost of interruptions

Proc. Power Engineering Society General Meeting, 2006.

40. California ISO, Integration of renewable resources: technical appendices for California ISO renewable integration studies version 1 [online] http://www.caiso.com/282d/282d85c9391b0.pdf

41. R. Billinton and R. Allan,

Reliability Evaluation of Power Systems,

Second Edition, London: Plenum Publishing Corporation, New York 1996.

42. L. Söder,

Simulation of wind speed forecast errors for operation planning of multiarea power systems,

Proc. Probabilistic Methods Applied to Power Systems, 2004 International Conference on, pp. 723-728, 12-16 Sep. 2004.

43. A. Boone,

Simulation of short-term wind speed forecast errors using a multi-variate ARMA $(1,1)$ time-series model

Master Thesis, KTH 2005.

44. J. F. Manwell, et al.

Hybrid2- a hybrid system simulation model theory manual

Renewable Energy Research Laboratory, Department of Mechanical Engineering, University of Massachusetts, Jun. 2006.

45. J. F. Manwell, J.G. McGowan, and A.L. Rogers,

Wind Energy Explained

Wiley, New York, 2002.

46. P. Glasserman,

Monte Carlo Method in Financial Engineering,

Springer, New York, 2003.

47. EATON Residential Smart Charging Solution [Online]. Available: http://www.eaton.com http://www.cleanfuelconnection.com/electric-vehicle-eaton-ev-chargers.html

\section{BIOGRAPHIES}

Ghazale Haddadian received the B.S. degree in computer software engineering from Sajad University of Mashhad, Iran, and the M.B.A. with dual concentration in finance and international business from Wright State University, Dayton, OH. She completed her Ph.D. degree in management science, with a concentration in operations management at Illinois Institute of Technology (IIT), Chicago. Presently she is an assistant professor in operations management at IIT.

Nasrin Khalili is an associate professor in Stuart School of Business at IIT. Professor Khalil has a B.S. in Chemistry and M.Sc and Ph.D. in Environmental Engineering and 8 years of experience in conducting research and teaching in the areas of environmental management and business decision-making. Her area of specialization ranges from synthesis and development of catalysts and adsorbents for removal of pollutants from industrial waste streams to analysis of environmental economics and cost valuations for sustainability projects, and development of energy portfolios models for rural areas.

Mohammad E. Khodayar received his B.S. degree from Amirkabir University of Technology, Tehran, Iran; M.S. degree from Sharif University of Technology, Tehran; and Ph.D. degree from IIT, Chicago, IL, USA, in 2012, all in electrical engineering. He was a Senior Research Associate with the Robert W. Galvin Center for Electricity Innovation at IIT. He is currently an assistant professor with the Department of Electrical Engineering, Southern Methodist University, Dallas, TX, USA. His current research interests include power system operation and planning.

Mohammad Shahidehpour is Bodine Chair Professor and Director of Robert W. Galvin Center for Electricity Innovation at IIT. Dr. Shahidehpour was the recipient of the Honorary Doctorate from the Polytechnic University of Bucharest in Romania. $\mathrm{He}$ is a research professor in a half dozen international universities, has published over 400 articles on power system operation and control and served as the founding Editor-in-Chief of the IEEE Transactions on Smart Grid. He is an IEEE Fellow. 